\title{
EnSuRe: Energy \& Accuracy Aware Fault-tolerant Scheduling on Real-time Heterogeneous Systems
}

\author{
Sangeet Saha ${ }^{1}$, Adewale Adetomi ${ }^{2}$, Xiaojun Zhai ${ }^{1}$, Server Kasap ${ }^{3}$, Shoaib Ehsan ${ }^{1}$, Tughrul Arslan ${ }^{2},{ }^{1}$ Klaus McDonald-Maier \\ ${ }^{1}$ Embedded and Intelligent Systems Laboratory, University of Essex, UK \\ ${ }^{2}$ Ewireless Research Group, School of Engineering, University of Edinburgh, UK \\ ${ }^{3}$ School of Computing, Electronics and Maths, Coventry University, UK \\ $\left\{{ }^{1}\right.$ sangeet.saha, ${ }^{1}$ xzhai, ${ }^{1}$ sehsan, $\left.{ }^{1} \mathrm{kdm}\right\} @$ @essex.ac.uk, $\left\{{ }^{2}\right.$ Adewale.Adetomi, ${ }^{2}$ T.Arslan $\} @$ ed.ac.uk , ${ }^{3}$ server.kasap@ coventry.ac.uk
}

\begin{abstract}
This paper proposes an energy efficient real-time scheduling strategy called EnSuRe, which (i) executes real-time tasks on low power consuming primary processors to enhance the system accuracy by maintaining the deadline and (ii) provides reliability against a fixed number of transient faults by selectively executing backup tasks on high power consuming backup processor. Simulation results reveal that $\mathrm{EnSuRe}$ consumes nearly 25\% less energy, compared to existing techniques, while satisfying the fault tolerance requirements. EnSuRe is also able to achieve $\mathbf{7 5 \%}$ system accuracy with $\mathbf{5 0 \%}$ system utilisation. Further, the obtained simulation outcomes are validated on benchmark tasks via a fault injection framework on Xilinx ZYNQ APSoC heterogeneous dual core platform.
\end{abstract}

Index Terms-Heterogeneous processors, Real-time systems, Fault-tolerant scheduling, Energy efficiency

\section{INTRODUCTION}

In real-time computing, correctness does not only depend on the precision of the results, but also on time at which these are produced. For such critical systems, approximated results obtained within the deadline are preferable over the accurate results generated after this deadline. In UAVs, initially an inaccurate, but acceptable quality image is generated from the received data. Then, based on the available resources and energy, the obtained image may be further refined [1]. Utilising approximate computation approaches, a real-time task consists of a mandatory part, followed by an optional part [2]. The mandatory part must be executed entirely in order to produce an acceptable result within a deadline, while the optional part will be executed for further refinement of the generated result and to provide a higher accuracy of the applications executed. However, as the mandatory parts have timing constraint, provisions must be made against faults. In order to handle these faults, typically tasks are re-executed on a backup processor to deliver the correct result [3].

In real-time scheduling, recently the authors in [4], [5], [6], have studied the combined problem of minimizing energy consumption while providing fault tolerance guarantees. However, these studies are limited to either uniprocessor systems or homogeneous multiprocessors. For heterogeneous systems, the authors in [3], [7], [8], have employed standby

This work is supported by the UK Engineering and Physical Sciences Research Council through grants EP/R02572X/1, EP/P017487/1 EP/V000462/1 and EP/V034111/1. sparing and primary/backup techniques to provide energy aware fault tolerant solutions. However these works consider hard real-time tasks, not emerging approximation based realtime tasks. Moreover, all of these studies employ standard scheduling scheme like Earliest-Deadline- First (EDF) and Earliest-Deadline-Late (EDL) scheduling policies. The authors also made a strict assumption that all tasks share a fixed and common deadline. In modern safety critical systems, such assumptions are no longer valid, because based upon their respective criticality, individual tasks must have unique deadlines. Thus, the proposed techniques may perform poorly on multiprocessor system, where multiple tasks require to complete execution requirements within multiple deadlines.

In this paper, given a set of real-time tasks to be executed on a heterogeneous multiprocessor system, we propose EnSuRe which i) tolerates total $k$ number of faults within the scheduling window ii) finishes task's execution within the deadlines and iii) enhances system accuracy while maintaining energy efficiency.

\section{Proposed ApproACH: EnSuRe}

We consider a real-time application $(\mathcal{A})$, which consists of a set of $n$ real-time tasks $T=\left\{T_{1}, T_{2}, \ldots, T_{n}\right\}$. Each task $T_{i}(1 \leq i \leq n)$ has a mandatory part, with an execution requirement of $M_{i}$ to be finished within the deadline, $d_{i}$ and an optional part with an execution requirement of $O_{i}$.

\section{A. Schedule generation phase}

EnSuRe employs a time-partitioning based [9] scheduling approach for a set of $n$ real-time tasks $\mathcal{A}=\left\{T_{1}, T_{2}, \ldots, T_{n}\right\}$ on the multiprocessor system. Let us consider the difference between any two consecutive deadlines (i.e. $\chi^{t h}$ and $(\chi-1)^{t h}$ task deadline) termed as "time window" and it can be found as:

$$
T W L_{\chi}=d_{\chi}-d_{\chi-1}
$$

For each task, the execution rate demand is denoted by its weight and can be found as: $w t_{i}=\frac{M_{i}}{d_{i}}$, where $M_{i}$ denotes the mandatory execution time and $d_{i}$ denotes the deadline. For any time-window each task will be allowed to be executed for a certain time span proportional to its weight and these spans 
for each individual task will be termed as "workload-quota" and can be calculated as:

$$
W Q u_{i}^{\chi}=\left(\left\lceil w t_{i} \times T W L_{\chi}\right\rceil\right) \quad \forall T_{i} \in \mathcal{A}
$$

Within a time-window, total system-wide capacity can be calculated as: $T W L_{\chi} \times m_{\text {pri }}$, where $m_{\text {pri }}$ is the number of available primary core. For a feasible schedule, this capacity must be equal or greater than the total workload-quota for all running tasks, i.e. $\left(\sum_{i=1}^{n} W Q u_{i}^{\chi}\right)$. Thus, the following condition must be satisfied to obtain a feasible schedule.

$$
\sum_{i=1}^{n} W Q u_{i}^{\chi} \leq T W L_{\chi} \times m_{p r i}
$$

EnSuRe will initiate the task allocation from the first primary core, as per their workload-quota. However, if the core is not completely occupied then the available slack $A S_{j}^{\chi}$ of the $j^{\text {th }}$ primary core for the $\chi^{\text {th }}$ time-window is:

$$
A S_{j}^{\chi}=T W L_{\chi}-\sum_{i=1}^{n} W Q u_{i}^{\chi} \times \theta_{i j}
$$

where $\theta_{i j}$ equals 1 if $T_{i}$ has been assigned to $j^{\text {th }}$ core; otherwise $\theta_{i j}=0$.

According to our strategy, this available slack will be used for the execution of optional portion of tasks so that the system accuracy can be enhanced. In order to allocate the optional portion of tasks within a time-window, we have defined a factor called "Urgency Factor (UF)", the urgency factor $\left(U F_{i}\right)$ of task $T_{i}$ can thus be defined as:

$$
U F_{i}=d_{i}-t^{\text {slack }}
$$

where $t^{\text {slack }}$ denotes the time instant where the slack time starts within a time-window.

\section{B. Fault handling phase}

After scheduling, EnSuRe creates a list called "backup" in non-increasing order of $M_{i}^{H P}$, where $M_{i}^{H P}$ denotes the execution length of mandatory part of $T_{i}$ on high performance backup core. As EnSuRe needs to handle only $k$ number of faults, it reserves an execution slot on HP for possible backup task execution. We termed this slot as "BES (Backup Execution Slot)". Then EnSuRe decides when to activate this "BES" slot inside a time-window. Thus, the "BST (Backup Start Time)" is calculated. If a the task is executed with zero error, then the result is committed. This in turn, removes the task from the backup list. Hence, as soon as a primary task completes successfully, the size of the "BES" slots on the HP core reduces dynamically. Algorithm 1 shows the pseudocode of EnSuRe.

\section{EXPERIMENTS AN ANALYSIS}

\section{A. Performance Metrics}

Normalized Energy Consumption (NEC) and Normalized Achieved Accuracy (NAA) have been used for evaluation. $N A A$ is the ratio between total executed optional portion and total available optional portions for all tasks.

The ranges of the mandatory portion $M_{i}$ and the optional portion $O_{i}$ are obtained from [2]. The weights $\left(w t_{i}=\frac{M_{i}}{d_{i}}\right)$ of

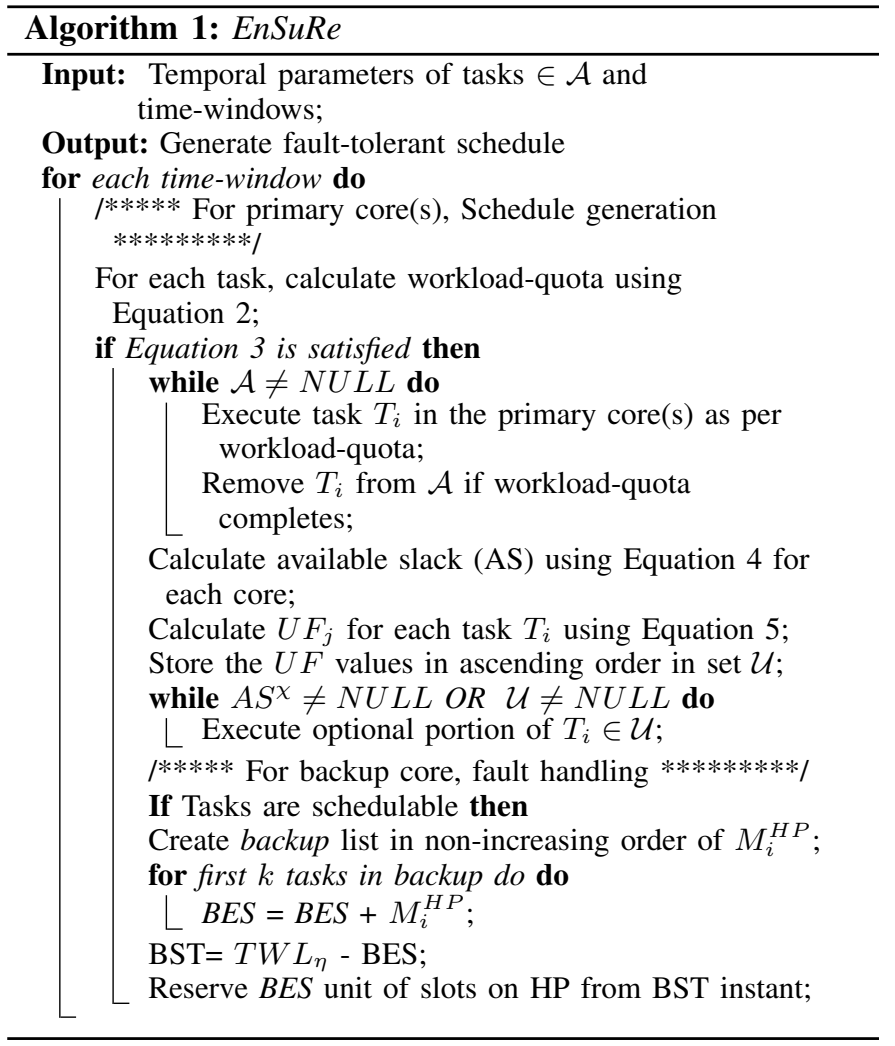

the tasks are generated from normal distribution with standard deviation $\sigma_{w t}=0.1$ and the mean values i.e. $\mu_{w t}=0.1$, $\mu_{w t}=0.2$. Having the tasks weights, the total workload of the system $\left(S y s_{W L}\right)$ can be obtained by adding the weights of all tasks. Hence, the system utilisation $\left(S y s_{u t i}\right)$ can be defined as:

$$
\text { Sys }_{u t i}=\frac{\text { Sys }_{W L}}{m_{\text {pri }}} \times 100 \%
$$

\section{B. Results and Analysis}

1) Evaluating the impact of $k$ : Figure 1(a) exhibits how energy consumption varies with increasing number of faults. As per the trends in Figure 1(a), it can be concluded that the higher the number of faults, the higher is the energy consumption for EnSuRe. However, SlowerP [7] consumes a fixed energy consumption. This behavior of SlowerP can be argued by the fact that irrespective number of faults, this strategy keeps a backup space for all tasks. In contrast, for EnSuRe as $k$ increases the BES also increases which in turn increases overall power consumption.

2) Evaluating the impact of utilisation: Figure 1(b) shows how the energy consumption varies with respect to varying system utilisation. The number of faults set as $k=4$. It may be observed from Figure 1(b) that with the increasing system utilisation, the energy consumption also increases for both EnSuRe and SlowerP. We have further compared EnSuRe with two existing strategies "LTF" and "TBLS" as proposed in [10]. "LTF" and "TBLS" gives higher priority to the tasks with higher execution length thus, in order to maintain deadline, the HP core is also used for primary execution which leads 


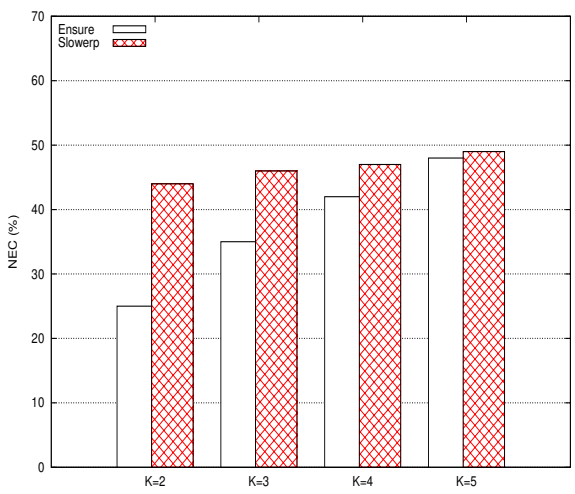

(a) Impact of number of faults

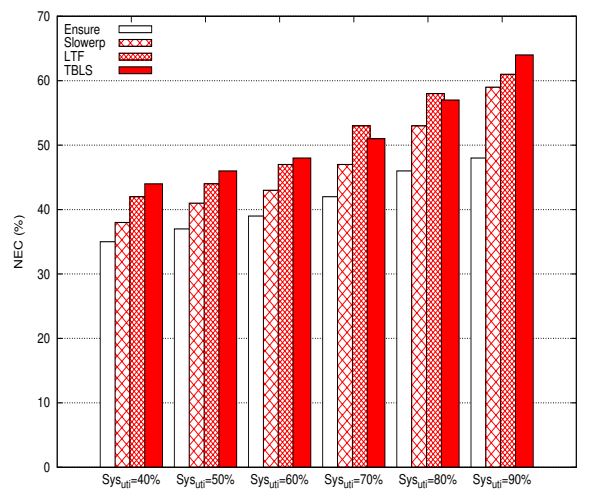

(b) Impact of system-utilisation

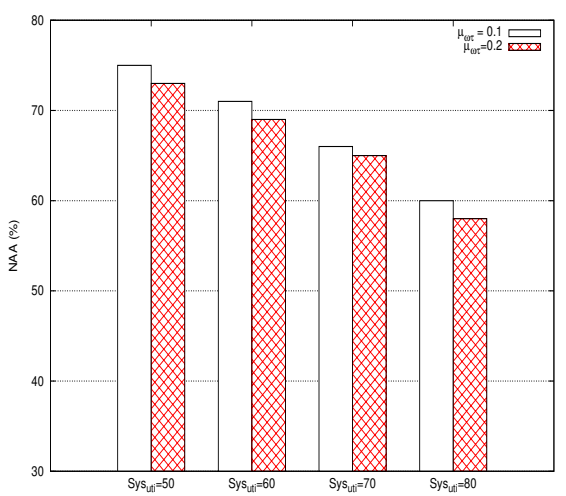

(c) NAA (\%) varying $\mu_{w t}$

Fig. 1: Performance of EnSuRe

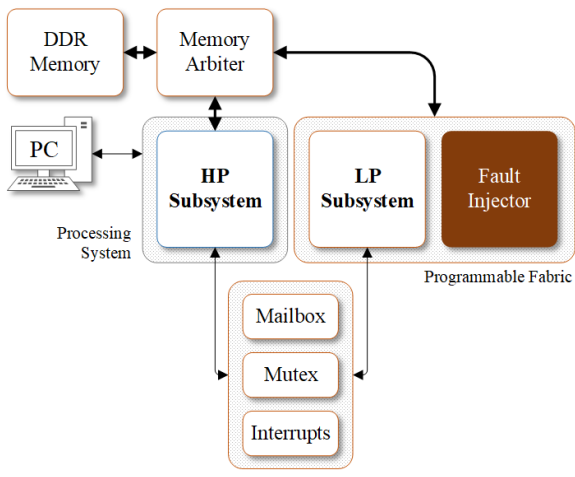

Fig. 2: The ZYNQ test-bed

to high energy consumption. It can be observed that in case of highest system utilisation (Sysuti $=90 \%$ ), EnSuRe consume $25 \%$ less energy than "TBLS".

From Figure 1(c), EnSuRe is able to achieve 75\% accuracy when Sys $_{u t i}$ is 50\%. However, as the utilisation increases the slack in primary core(s) decreases and thus, NAA decreases with the increase in $S y s_{u t i}$. It has to be noted that for a $S y s_{u t i}$, if the $\mu_{w t}$ varies from 0.1 to 0.2 , the NAA remains comparable.

\section{HARDWARE IMPLEMENTATION}

\section{A. Architectural Setup}

We have implemented EnSuRe on a heterogeneous system on a Xilinx Zynq-7000 All-Programmable SoC [11], with Arm Cortex-A9 CPU in the Processing System (PS) side, which serves as the HP core; and FPGA fabric in the Programmable Logic (PL) side, which is used to implement the LP core and other system components. Figure 2 represents the proposed architecture. The host PC executes the EnSuRe algorithm.

\section{B. Fault Injection and Detection Framework}

The fault injection framework needed to confirm the integrity of the TMR MicroBlaze Subsystem relies on the TMR Inject IP core. Fault injection is actually carried out by injecting a different instruction at a certain instruction address of one of the three processors. This causes a mismatch among the processors and such mismatch is detected by a TMR comparator. The framework is shown in Figure 3.

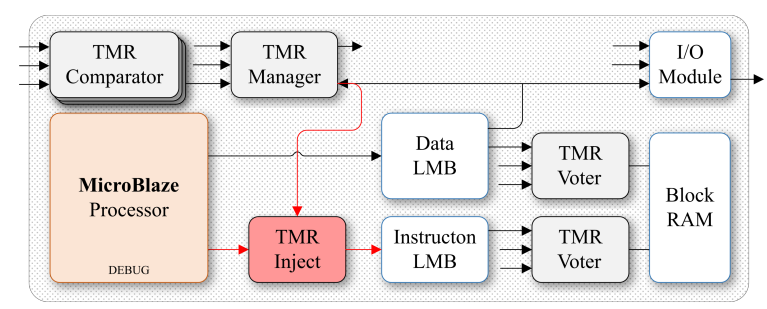

Fig. 3: Fault injection and detection

\section{Energy consumption}

We have created synthetic tasks from MiBench benchmark [2]. The execution times for HP core and LP core are measured for ARM core (freq: $650 \mathrm{MHz}$ ) and MicroBlaze core (freq: $100 \mathrm{MHz}$ ). We have evaluated the EnSuRe by injecting $(k=3)$ faults.The average scheduling length is taken as 30000 ms and we executed the simulations 5 times by injecting the faults at arbitrary positions in the scheduling length. The final value is calculated from the average of these obtained values. Table I shows the energy consumption of EnSuRe and SlowerP for the entire scheduling length.

TABLE I: Enrgy Consumption in Joule

\begin{tabular}{|c|c|c|}
\hline Avg. number of tasks & EnSuRe & SlowerP \\
\hline 8 & 7.83 & 11.26 \\
\hline 12 & 9.68 & 14.57 \\
\hline 16 & 13.58 & 17.84 \\
\hline
\end{tabular}

\section{CONClusion}

EnSuRe schedule tasks on primary core such that tasks could meet their deadlines and accuracy can be enhanced. However, the backup core is intelligently used for energy efficiency and fault tolerance. As per the obtained simulation behavior, it can be argued that EnSuRe can be employed for energy efficient operation and the simulation outcomes are further validated on ZYNQ APSoC heterogeneous systems with benchmark tasks. 


\section{REFERENCES}

[1] S. Mittal, "A survey of techniques for approximate computing," ACM Computing Surveys (CSUR), vol. 48, no. 4, p. 62, 2016.

[2] L. Mo, A. Kritikakou, and O. Sentieys, "Approximation-aware task deployment on asymmetric multicore processors," in 2019 Design, Automation \& Test in Europe Conference \& Exhibition (DATE). IEEE, 2019, pp. 1513-1518.

[3] Y. Guo, D. Zhu, H. Aydin, J.-J. Han, and L. T. Yang, "Exploiting primary/backup mechanism for energy efficiency in dependable realtime systems," Journal of Systems Architecture, vol. 78, pp. 68-80, 2017.

[4] M. A. Haque, H. Aydin, and D. Zhu, "On reliability management of energy-aware real-time systems through task replication," IEEE Transactions on Parallel and Distributed Systems, vol. 28, no. 3, pp. 813-825, 2016

[5] M. Fan, Q. Han, and X. Yang, "Energy minimization for on-line realtime scheduling with reliability awareness," Journal of Systems and Software, vol. 127, pp. 168-176, 2017.

[6] B. Zhao, H. Aydin, and D. Zhu, "Energy management under general task-level reliability constraints," in 2012 IEEE 18th Real Time and Embedded Technology and Applications Symposium. IEEE, 2012, pp. 285-294.

[7] A. Roy, H. Aydin, and D. Zhu, "Energy-aware standby-sparing on heterogeneous multicore systems," in 2017 54th ACM/EDAC/IEEE Design Automation Conference (DAC). IEEE, 2017, pp. 1-6.

[8] P. P. Nair, R. Devaraj, and A. Sarkar, "Fest: Fault-tolerant energyaware scheduling on two-core heterogeneous platform," in 2018 8th International Symposium on Embedded Computing and System Design (ISED). IEEE, 2018, pp. 63-68.

[9] S. Saha, X. Zhai, S. Ehsan, S. Majeed, and K. McDonald-Maier, "Rasa Reliability-aware scheduling approach for fpga-based resilient embedded systems in extreme environments," IEEE Transactions on Systems, Man, and Cybernetics: Systems, 2021.

[10] A. Roy, H. Aydin, and D. Zhu, "Energy-efficient fault tolerance for real-time tasks with precedence constraints on heterogeneous multicore systems," in 2019 Tenth International Green and Sustainable Computing Conference (IGSC). IEEE, 2019, pp. 1-8.

[11] L. Crockett, D. Northcote, C. Ramsay, F. Robinson, and R. Stewart, $E x$ ploring Zynq MPSoC: With PYNQ and Machine Learning Applications, 2019. 